\title{
Diurnal variation in corticosterone release among wild tropical forest birds
}

Philipp Schwabl ${ }^{1 *}$, Elisa Bonaccorso ${ }^{2}$ and Wolfgang Goymann ${ }^{3}$

\begin{abstract}
Background: Glucocorticoids are adrenal steroid hormones essential to homeostatic maintenance. Their daily variation at low concentrations regulates physiology and behavior to sustain proper immunological and metabolic function. Glucocorticoids rise well above these baseline levels during stress to elicit emergency-state responses that increase short-term survival. Despite this essence in managing life processes under both regular and adverse conditions, relationships of glucocorticoid release to environmental and intrinsic factors that vary at daily and seasonal scales are rarely studied in the wild.

Methods: This study on 41 passerine species of the Ecuadorian Chocó applied a standardized capture-and-restraint protocol to examine diurnal variation in baseline and stress-related release of corticosterone, the primary avian glucocorticoid. Tests for relationships to relative body mass, hemoglobin concentration, molt status and date complemented this evaluation of the time of day effect on corticosterone secretion in free-living tropical rainforest birds. Analyses were also partitioned by sex as well as performed separately on two common species, the wedgebilled woodcreeper and olive-striped flycatcher.

Results: Interspecific analyses indicated maximum baseline corticosterone levels at the onset of the active phase and reductions thereafter. Stress-related levels did not correspond to time of day but accompanied baseline reductions during molt and elevations in birds sampled later during the September - November study period. Baseline corticosterone related negatively to hemoglobin in the wedge-billed woodcreeper and stress-related levels increased with body mass in the olive-striped flycatcher. There were no substantial sex-related differences.

Conclusions: The results of this study suggest a diurnal rhythmicity in baseline corticosterone release so robust as to emerge in pooled analyses across a highly variable dataset. While this detection in nature is singular, correspondent patterns have been demonstrated outside of the tropics in captive model species. Congruity in daily rhythms and links to physiological and life-history state across disparate taxa and environments may promote the yet unresolved utility of corticosterone release as a global metric for population health. However, certain results of this study also deviate from laboratory and field research at higher latitudes, cautioning generalization. Environmental distinctions such as high productivity and tempered seasonality may precipitate unique life-history strategies and underlying hormonal mechanisms in tropical rainforest birds.
\end{abstract}

Keywords: Corticosterone, Birds, Tropical, Diurnal rhythms, Daily variation, Free-living, Wild, Chocó, Stress

\footnotetext{
* Correspondence: p.schwabl.1@research.gla.ac.uk

'Institute of Biodiversity, Animal Health and Comparative Medicine, University

of Glasgow, Graham Kerr Building, Glasgow G12 8QQ, UK

Full list of author information is available at the end of the article
} 


\section{Background}

Glucocorticoids are adrenal steroid hormones integral to physiological and behavioral control in nearly all vertebrate animals. Circulating at low levels in the blood, they serve preeminently as regulators of basic energy balance, directing resource acquisition, deposition and mobilization [1]. Upon perception of a stressor, however, glucocorticoid levels rapidly rise and increase short-term survival probability by eliciting increased cardiovascular tone, hyperglycemia, modified immune activity and enhanced memory consolidation (etc.) while diverting effort from nonessential life processes such as reproduction [2, 3].

Diel rhythms in glucocorticoid activity have been established in mammals and are particularly well characterized in rodent model species [4, 5]. Such rhythms have also been detected early on in birds [6], but consensus here remains rudimentary [7, 8]. Most studies agree in little more than that baseline corticosterone, the primary avian glucocorticoid [9], tends to circulate at maximum levels in early morning hours, often at or just prior to the onset of diurnal activity [6,10-15]. Even less agreement has taken form on diel rhythms in avian stressrelated corticosterone release. Only three avian species have been examined deliberately for diel variation in this trait, with maximum stress-related levels found during the inactive phase in house sparrows [16] and starlings [13]. In contrast, Gambel's white-crowned sparrows show maximum levels during the active phase [12]. Discord extends also to data on modulation by environmental and intrinsic factors such as photoperiod (e.g. photoperiod-dependent adrenal sensitivity in house sparrows [8] vs. independent responses in starlings [13]) and physiological state (e.g. unexhausted [17] vs. maximally stimulated adrenal capacity to secrete corticosterone in molting house sparrows [8]).

This elementary understanding of rhythmic avian adrenocortical function stems primarily from experimental studies on captive birds $[14,18]$. However, it is recurrently emphasized that data from captivity can only partially represent wild avian corticosterone secretion [8, $19,20]$, including its diel rhythmicity $[12,19]$. Not only are few avian species amenable to laboratory experimentation (with bias towards poultry and other domesticated species $[14,18])$, a diminishment of environmental and social cues constrains the range of hormone levels exhibited in captivity [21] and may mask diel change [12].

Field data on avian corticosterone release are particularly lacking for near-equatorial species [22]. Past studies have heavily favored the temperate and arctic zone [23, 24]. In contrast, variation in life-history characters has been mapped much more comprehensively across latitudes and distinctions in several of these traits (e.g. incubation period and clutch size) have been established for the tropics [25]. Given that endocrine mechanisms mediate life-history trade-offs [26], corresponding geographical variation in corticosterone release is likely and merits exploration [22, $27,28]$. For example, as low levels of yolk androgens are coupled to long incubation periods in the tropics $[29,30]$, low levels of parental baseline corticosterone may relate to small clutches reared here over longer breeding seasons $[31,32]$. Implications for conservation biology also promote intensified field research on tropical avian corticosterone release. Adrenocortical performance has become an increasingly popular biomarker of population health [1] However, widespread dispute over its diagnostic utility and superiority to traditional demographic methods must first be resolved [33-35] with enhanced comprehension of how physiological condition and fitness relate to corticosterone secretion in the wild. These relationships are likely specific and distinct to different environments [33, 36, 37]. It is therefore sensible to prioritize further research on corticosterone release to the most vulnerable and biodiverse regions of the world. The tropical Andes are one such hotspot of conservation priority [38].

In this study we describe diurnal variation in baseline and stress-related corticosterone release in free-living individuals of 41 passerine species of northwest Ecuador. Our results on a diverse array of forest birds of the tropical Andes should forward understanding in a field deficient of data on passerines [16] and from wild birds in general [39], specifically regarding diel patterns of stress-related corticosterone release. We also evaluate the time of day effect on corticosterone variation against the additional predictors relative body mass, hemoglobin concentration, molt status and sampling date in our models. These physiological and seasonal variables represent two prominent correlates of avian corticosterone secretion - body condition [40] and lifehistory stage [24], the former of which often co-varies with time of day [41]. As such, our study contributes data required to resolve geographic and taxonomic differences in short and long-term avian adrenocortical activity essential to conservation decisions for the Ecuadorian Chocó, one of the most biodiverse regions of the world [42].

\section{Methods \\ Study area}

A total of 169 individuals comprising 55 passerine bird species were captured daily between 23 September and 22 November 2014 at Mashpi Rainforest Biodiversity Reserve $\left(0^{\circ} 9^{\prime} \mathrm{N}, 78^{\circ} 51^{\prime} \mathrm{W}\right)$ in the Chocó bioregion of Ecuador near Pacto, Pichincha Province. Rainfall is high throughout the year at this reserve. There are no distinct wet and dry seasons, but interpolated climate records specify April as the rainiest month and place a minimum on July [43]. Sunrise and sunset are medially at 5:56 h and 18:03 $\mathrm{h}$ at the site [44]. The sampling period coincided in large part with local breeding activity, as determined morphometrically and based on observations of parental behavior. Although avian breeding phenology remains enigmatic 
and is unlikely discriminable by region or taxon, corresponding reproductive peaks during the period between September and November have been documented elsewhere in the Ecuadorian Chocó [45-47]. Three of the six sampling sites selected were located at $600 \mathrm{~m}$ elevation, separated from one another by ca. $1 \mathrm{~km}$, and three sites were located at $1200 \mathrm{~m}$ elevation, also separated by ca. $1 \mathrm{~km}$ each. All sites consisted of secondary-growth humid montane forest and were selected based on equable proximity to forest edges, streams and human activity.

\section{Capture and sampling protocol}

Birds were captured predominantly by passive mist-netting (and in two cases using conspecific playback recorded on site). Nets remained under constant surveillance from 6:00 to $16: 30 \mathrm{~h}$ such that a standardized stress protocol could be applied immediately upon capture. As corticosterone levels may become elevated in the blood within 3 min following a stress stimulus [48], initial blood samples to determine baseline hormone concentrations were collected as quickly as possible via puncture of the brachial vein and capillary withdrawal into heparinized micro-hematocrit tubes. Latency time between a bird's first contact with the net and withdrawal of ca. $40 \mu \mathrm{l}$ blood was recorded $($ median $=2.8 \mathrm{~min})$ and did not exceed $3 \mathrm{~min}$ for 93 of 95 samples submitted to laboratory analysis. Birds were then given a numbered color band, weighed to $0.1 \mathrm{~g}$ by digital scale (American Weigh Scales, Inc.), measured for tarsus length to $0.1 \mathrm{~mm}$ by digital caliper (Mitutoyo America Corporation) and assessed for pre-basic molt in remiges, rectrices and feathers of the head, chest, abdomen and back. Reproductive characters (brood patch and cloacal protuberance) served to assign sex in the absence of plumage dimorphism. Sex was left undetermined for 34 monomorphic individuals. Birds were then placed in an opaque cloth bag until $30 \mathrm{~min}$ postcapture, at which time a second, slightly smaller blood sample $($ ca. $30 \mu \mathrm{l})$ was withdrawn to determine acute stressrelated (standard bag-restraint) corticosterone release. At this time, a small (ca. $2 \mu \mathrm{l}$ ) aliquot of blood was also assessed for hemoglobin concentration (g/l) with a portable photometric hemoglobinometer (EKF Diagnostic). Plasma for determination of baseline and stress-related corticosterone release was separated from the blood cell fraction immediately after each withdrawal using a battery-powered mini-centrifuge (LW Scientific, Inc.) and then transferred to $250 \mu \mathrm{l}$ vials. Vials were kept on ice in a portable cooler until the end of each day and then stored frozen at $-20^{\circ} \mathrm{C}$.

\section{Corticosterone analysis}

Corticosterone concentrations of 190 plasma samples from 95 individuals comprising 41 species were determined by direct radioimmunoassay (RIA) following [49] and outlined below. As referenced above, this subset was selected based on latency time of the first blood withdrawal (for veracious measurement of baseline corticosterone), with analysis completed in three tiered extraction/assay procedures.

To extract corticosterone, measured plasma volumes $($ mean $=22 \mu \mathrm{l})$ were first vortexed with $10 \mu \mathrm{l}(1500 \mathrm{dpm})$ labelled corticosterone overnight at $4{ }^{\circ} \mathrm{C}$ to allow for equilibration with plasma lipids and binding proteins. Steroid isolation followed by repeated dichloromethane extraction, in which freshly distilled dichloromethane was added to the centrifuged plasma samples, the aqueous phase fixed by freezing in pure ethanol and dry ice and the organic phase decanted and evaporated with nitrogen in a $40{ }^{\circ} \mathrm{C}$ water bath. The corticosterone sediment was then left to equilibrate overnight at $4{ }^{\circ} \mathrm{C}$ in $300 \mu \mathrm{l} 0.1 \mathrm{M}$ neutral-pH phosphate-buffered saline (with $1 \%$ gelatin-PBSG). To determine extraction efficiency, an $80 \mu \mathrm{l}$ aliquot was then drawn from the corticosteronebuffer solution, mixed with $4 \mathrm{ml}$ scintillation fluid (Packard Ultima Gold) and quantified for $\beta$-radiation in a Beckman LS 6000 counter alongside total recovery counts prepared prior to extraction.

In proceeding to RIA, each vortexed sample was partitioned into $100 \mu \mathrm{l}$ duplicates. Positive $(25 \mu \mathrm{l}$ corticosterone standard and $100 \mu \mathrm{l}$ chicken plasma controls) and negative controls (blanks) were also prepared. The standard curve was generated by serial dilution of $100 \mu \mathrm{l}$ unlabeled corticosterone in $100 \mu \mathrm{l}$ PBSG, effectively halving concentrations in each dilution step to generate ten $100 \mu \mathrm{l}$ standard duplicates ranging from 1000 to $1.95 \mathrm{pg}$ steroid content. Sample duplicates, standard curve duplicates, and controls were then mixed with $100 \mu \mathrm{l}$ corticosterone antiserum and, after $30 \mathrm{~min}$, treated with $100 \mu \mathrm{l}$ labelled steroid. In addition to the curve standards, TC $(100 \mu \mathrm{l}$ labelled steroid and $200 \mu \mathrm{l}$ PBSG), NSB (likewise $100 \mu \mathrm{l}$ labelled steroid and $200 \mu \mathrm{l}$ PBSG) and $B_{0}(100 \mu l$ labelled steroid, PBSG and antiserum) were prepared in $300 \mu$ l triplicates to determine total counts, non-specific (background) binding and maximum binding with the antiserum, respectively. All solutions were then left to incubate at $4{ }^{\circ} \mathrm{C}$ for $20 \mathrm{~h}$. Finally, bound and free corticosterone was separated at $4{ }^{\circ} \mathrm{C}$ via addition of $500 \mu \mathrm{l}$ dextran-coated charcoal to adsorb all unbound steroid, centrifuged under cooling $\left(4{ }^{\circ} \mathrm{C}\right)$ for $10 \mathrm{~min}$ at $4000 \mathrm{rpm}$, decanted into scintillation vials, mixed with $4 \mathrm{ml}$ scintillation fluid and quantified for $\beta$-radiation in the scintillation counter.

Standard curves and endogenous corticosterone concentrations were calculated with Immunofit 3.0 (Beckman Coulter, Inc.). For the three assays, mean ( \pm sd) corticosterone extraction recovery was $88.0 \pm 3.0 \%, 86.8 \pm 3.4 \%$ and $85.0 \pm 3.6 \%$. Lower detection limits were $32.6 \mathrm{pg} / \mathrm{ml}$, $31.2 \mathrm{pg} / \mathrm{ml}$ and $30.6 \mathrm{pg} / \mathrm{ml}$, respectively, and all samples were above these limits. The intra-assay coefficients of 
variation were $5.3 \%, 4.4 \%$ and $8.1 \%$, and the intraextraction coefficients of variation of a chicken plasma pool were $7.6 \%, 9.7 \%$ and $6.8 \%$, respectively. The inter-assay coefficient of variation among the three assays was $3.5 \%$ and their inter-extraction coefficient of variation was $4.3 \%$.

\section{Statistical procedures}

Statistical analyses were performed in $\mathrm{R}$ version 3.2.3 [50]. To assess diurnal trends in baseline and stressrelated corticosterone release, means from birds captured during 6:09 h-8:50 h, 8:51 h-11:06 h, 11:07 h12:49 $\mathrm{h}$ and 12:50 $\mathrm{h}-16: 23 \mathrm{~h}$ sampling time periods were first compared by one-way analyses of variance (ANOVA). These 4 categories were chosen to partition the daily capture period into sequential time intervals comprising equal sample sizes $(n=23,23,23$ and 24, respectively) of sufficient statistical power. Each of these intervals incidentally holds one of four bouts of avian activity observed to correspond with daily weather trends at the study site. Birds generally arrived at capture sites discontinuously as members of foraging flocks. These mixed groups often peaked in activity near 8:00 $\mathrm{h}$ as rains subsided and again around 12:00 $\mathrm{h}$ before winds and temperatures rose into afternoon. A third peak recurred in the hour before dusk. 9:00 h-11:00 h also often coincided with elevated activity under overcast, temperate conditions. Results from the categorization scheme outlined above were then validated by sorting data into four sequential intervals of equal length in time (158 min each) and reiterating ANOVA for differences in mean baseline and stress-related corticosterone release. For inferences from the models we obtained Bayesian posterior parameter estimates and their $95 \%$ credible intervals, employing the $\operatorname{sim}$ function in the arm package [51] over 10,000 simulations with an uninformed prior distribution. Unlike null-hypothesis testing, Bayesian statistics do not provide $p$-values. Instead, meaningful differences between groups are assessed by comparing ranges of $95 \%$ credible intervals. These intervals provide an estimate of 0.95 probability for the group means. If the credible interval of one group does not overlap with the mean estimate of another group, the groups are assumed to differ from each other.

To further evaluate baseline and acute stress-related corticosterone dependence, separate linear regression models were fitted to the corticosterone response variables to test the categorical predictor molt status and the covariates sampling time of day, sampling date, sampling latency, hemoglobin concentration and body mass : tarsus ratio. The latter ratio was replaced by a scaled mass index [52] in intraspecific analyses for which additional mass and tarsus measurements were available from prior studies at the site (unpublished data from C. Morochz). This index may more reliably indicate size of energy reserves and other components of body condition in birds. Values for all covariates were standardized by centering (subtraction of the sample mean) and scaling (division by the sample standard deviation) to z-scores. Regression models were selected by ranking candidate models incorporating one to all combinations of the independent variables by Akaike's information criterion modified for small sample size $\left(\mathrm{AIC}_{\mathrm{c}}\right.$ [53]). As this criterion includes a penalty for each incorporated parameter, encounters with chance correlations by multiple testing were restrained by the method's direction to balance model fit and size. Substantial support was inferred only for models within $2 \mathrm{AIC}_{\mathrm{c}}$ of the first-ranked model (i.e. $\Delta \mathrm{AIC}_{\mathrm{c}} \leq 2$ ) and for which credible intervals of all predictor coefficients did not overlap zero. Retained models were then checked for normality of the error distribution, homoscedasticity of the errors, leverage and linearity in the relationship between predictor and response variables. In consequence, ANOVA and model selection programs were reiterated applying logtransformed response variables.

The $\mathrm{AIC}_{\mathrm{c}}$ model selection procedures outlined above were first performed across all species to investigate general patterns. Within this interspecific tier of analysis, separate models for males and females were also established. Intraspecific analyses followed for the dataset's best sampled species, the wedge-billed woodcreeper (Glyphorynchus spirurus; $n=17$ ) and the olive-striped flycatcher (Mionectes olivaceous; $n=10$ ). Sex was not partitioned in these intraspecific analyses due to limited sample sizes. In all analyses, baseline corticosterone concentrations were applied directly while acute stressrelated changes in corticosterone were applied as relative increases from the baseline concentration: (30 min postcapture corticosterone concentration) - (baseline corticosterone concentration). This latter variable was also applied directly as absolute (gross, independent of baseline) concentrations measured $30 \mathrm{~min}$ post-capture, because baseline and stress-related concentrations of corticosterone may be independent and regulate different traits $[3,17]$. This measure may additionally serve to rate maximum stress-related corticosterone release capacity and is hereafter offset with quotation marks and termed "stress-induced corticosterone" (in distinction to the relative measure "increase in corticosterone", also specified in quotes hereafter). Given its independence to baseline concentrations, modeling of "stress-induced corticosterone" disregarded sampling latency as a candidate variable. Accordingly, omission of data from two individuals (one orange-bellied euphonia, Euphonia xanthogaster, and one slaty-capped flycatcher, Leptopogon superciliaris) sampled for baseline corticosterone later than $3 \mathrm{~min}$ post-capture $(3.5 \mathrm{~min}$ and $4 \mathrm{~min}$ latency, respectively) was necessary only in analyses of baseline and "increase in corticosterone", not in analysis of "stress- 
induced corticosterone". Lastly, the ape package [54] in R was employed to establish a strict consensus phylogenetic tree from recent avian molecular phylogenies [55] for the species of this study.

\section{Results}

Baseline corticosterone concentrations differed among sampling intervals (ANOVA, $F_{3,89}=4.427$ ), with levels obtained from the first (early morning) and second (late morning) sampling intervals higher than those from the third (mid-day) and fourth (afternoon) intervals. This change in baseline corticosterone over time intervals chosen to aggregate data by equal sample size remained when arranging data for equal length in time (ANOVA, $\left.F_{3,89}=4.611\right)$. Again, baseline concentrations from the first and second of these equal-length $(158 \mathrm{~min})$ intervals were higher than those from the third and fourth such intervals (see Fig. 1 for Bayesian posterior mean estimates and credible intervals). Sampling time of day also correlated with baseline corticosterone levels in subsequent linear regression analysis (see below). "Increase in corticosterone" and "stress-induced corticosterone" did not vary over the day's sampling intervals.

$\mathrm{AIC}_{\mathrm{c}}$ model comparison (Table 1 and Additional file 1: Table S1) identified molt status, sampling time of day and sampling latency as the best predictors of interspecific variation in baseline corticosterone $\left(r^{2}=0.29, \mathrm{~F}_{3,89}\right.$ $=12.010)$. Molting individuals and those sampled later in the day yielded lower baseline levels than non-molting individuals and those sampled earlier in the day. Baseline levels increased with sampling latency (even within the 3 min latency cut-off applied to select samples). In interspecific analysis partitioned by sex, the model selected for males retained the effect of these three predictors yet additionally identified sampling date as a fourth explanatory variable $\left(r^{2}=0.49, \mathrm{~F}_{4,25}=6.044\right)$, with individuals sampled later in the September - November study period yielding higher levels of baseline corticosterone. Baseline levels in females were also best explained by a negative relationship to the predictors molt status and sampling time of day $\left(r^{2}=0.29, F_{2,26}=5.234\right)$, yet without correlation to sampling latency or sampling date. No model for sex-independent interspecific variation in "increase in corticosterone" met selection criteria. In sexdependent analyses, however, molt status and sampling date best predicted male "increase in corticosterone" $\left(r^{2}\right.$ $=0.27, F_{2,27}=9.949$ ), with molting individuals and those sampled at earlier dates showing lower increases than non-molting individuals and those sampled at later dates. Within females, substantial support was not found for any model of "increase in corticosterone", although the top-ranked model figured a positive trend to sampling date $\left(r^{2}=0.14, \mathrm{~F}_{1,26}=4.058\right)$. Sex-independent interspecific variation in "stress-induced corticosterone" was best albeit insubstantially explained by molt status $\left(r^{2}=0.06, \mathrm{~F}_{1,93}=5.526\right)$, with molting individuals yielding lower levels $30 \mathrm{~min}$ post-capture than non-molting individuals. In male-specific analysis, sampling date complemented the negative relationship to molt to explain interspecific "stress-induced corticosterone" more substantially $\left(r^{2}=0.26, \mathrm{~F}_{2,28}=4.971\right)$, with individuals sampled at later dates showing higher levels after $30 \mathrm{~min}$ than those sampled at earlier dates. Within females, substantial support was not found for any model of "stressinduced corticosterone".

Intraspecific model comparison identified hemoglobin concentration and sampling time of day as best predictors of baseline corticosterone in the wedge-billed

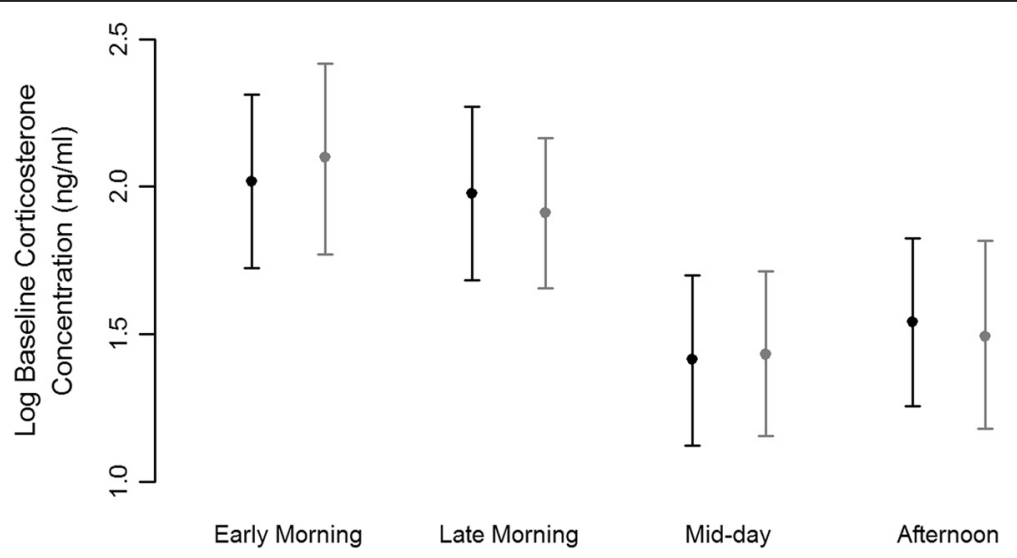

Fig. 1 Posterior means of baseline corticosterone measured in blood samples from birds captured at different times of day. Black points denote time intervals selected to partition data into sets of equal sample size $(n=23,23,23$ and 24, respectively). Grey points sort data into sets of equal length in time (i.e. 4 sequential intervals of 158 min each; $n=19,30,25$ and 19). Dispersion bars indicate $95 \%$ credible intervals and suggest corticosterone levels obtained from the first sampling interval $(2.018 \mathrm{ng} / \mathrm{ml}$ and $2.099 \mathrm{ng} / \mathrm{ml})$ to be higher than those from the third interval $(1.417 \mathrm{ng} / \mathrm{ml}$ and $1.434 \mathrm{ng} / \mathrm{ml})$. Dispersion of posterior means from the second $(1.977 \mathrm{ng} / \mathrm{ml}$ and $1.911 \mathrm{ng} / \mathrm{ml})$ and fourth intervals $(1.541 \mathrm{ng} / \mathrm{ml}$ and $1.494 \mathrm{ng} / \mathrm{ml}$ ) overlap slightly with others. Log denotes the natural logarithm (to base e) 
Table 1 Baseline corticosterone $\left(y_{1}\right)$, "increase in corticosterone" $\left(y_{2}\right)$ and "stress-induced corticosterone" ( $\left.y_{3}\right)$ models selected from $\mathrm{AIC}_{\mathrm{C}}$ analyses

\begin{tabular}{|c|c|c|c|c|c|c|c|c|c|}
\hline & \multirow{2}{*}{$\begin{array}{l}\text { Response } \\
\text { Variable }\end{array}$} & \multicolumn{7}{|c|}{ Linear Formula (Intercept and Variable Coefficients) } & \multirow[t]{2}{*}{$\omega_{\mathrm{i}}$} \\
\hline & & Int. & Time & Date & Molt & Hemo. & R. Mass & Lat. & \\
\hline \multirow[t]{2}{*}{ Total } & $y_{1}$ & $\begin{array}{l}+1.871 \\
{[+1.697,+2.046]}\end{array}$ & $\begin{array}{l}-0.236 \\
{[-0.388,-0.086]}\end{array}$ & - & $\begin{array}{l}-0.325 \\
{[-0.631,-0.016]}\end{array}$ & - & & $\begin{array}{l}+0.296 \\
{[+0.150,+0.444]}\end{array}$ & 0.29 \\
\hline & $y_{3}$ & $\begin{array}{l}+4.130 \\
{[+3.976,+4.283]}\end{array}$ & - & - & $\begin{array}{l}-0.310 \\
{[-0.575,-0.048]}\end{array}$ & - & - & $\mathrm{n} / \mathrm{a}$ & 0.19 \\
\hline \multirow[t]{3}{*}{ Males } & $y_{1}$ & $\begin{array}{l}+2.179 \\
{[+1.846,+2.514]}\end{array}$ & $\begin{array}{l}-0.312 \\
{[-0.549,-0.075]}\end{array}$ & $\begin{array}{l}+0.377 \\
{[+0.095,+0.646]}\end{array}$ & $\begin{array}{l}-0.714 \\
{[-1.324,-0.104]}\end{array}$ & - & - & $\begin{array}{l}+0.278 \\
{[+0.026,+0.525]}\end{array}$ & 0.26 \\
\hline & $y_{2}$ & $\begin{array}{l}+4.303 \\
{[+3.963,+4.640]}\end{array}$ & - & $\begin{array}{l}+0.376 \\
{[+0.076,+0.676]}\end{array}$ & $\begin{array}{l}-0.838 \\
{[-1.436,-0.259]}\end{array}$ & - & - & - & 0.24 \\
\hline & $y_{3}$ & $\begin{array}{l}+4.396 \\
{[+4.087,+4.716]}\end{array}$ & - & $\begin{array}{l}+0.378 \\
{[+0.094,+0.665]}\end{array}$ & $\begin{array}{l}-0.757 \\
{[-1.319,-0.208]}\end{array}$ & - & - & $\mathrm{n} / \mathrm{a}$ & 0.36 \\
\hline Females & $y_{1}{ }^{a}$ & $\begin{array}{l}+1.838 \\
{[+1.491,+2.198]}\end{array}$ & $\begin{array}{l}-0.356 \\
{[-0.641,-0.064]}\end{array}$ & - & $\begin{array}{l}-0.661 \\
{[-1.204,-0.108]}\end{array}$ & - & - & - & 0.09 \\
\hline \multirow[t]{2}{*}{$\begin{array}{l}\text { G. } \\
\text { spirurus }\end{array}$} & $y_{1}$ & $\begin{array}{l}+1.952 \\
{[+1.591,+2.312]}\end{array}$ & $\begin{array}{l}-0.667 \\
{[-1.110,-0.219]}\end{array}$ & - & - & $\begin{array}{l}-0.713 \\
{[-1.187,-0.235]}\end{array}$ & - & - & 0.36 \\
\hline & $y_{3}$ & $\begin{array}{l}+3.525 \\
{[+3.171,+3.863]}\end{array}$ & - & $\begin{array}{l}-0.439 \\
{[-0.842,-0.042]}\end{array}$ & - & - & - & $n / a$ & 0.21 \\
\hline \multirow[t]{2}{*}{$\begin{array}{l}\text { M. } \\
\text { olivaceus }\end{array}$} & $y_{2}$ & $\begin{array}{l}+4.510 \\
{[+4.218,+4.805]}\end{array}$ & - & - & - & - & $\begin{array}{l}+0.366 \\
{[+0.057,+0.682]}\end{array}$ & - & 0.51 \\
\hline & $y_{3}$ & $\begin{array}{l}+4.601 \\
{[+4.318,+4.885]}\end{array}$ & - & - & - & - & $\begin{array}{l}+0.346 \\
{[+0.048,+0.639]}\end{array}$ & $\mathrm{n} / \mathrm{a}$ & 0.54 \\
\hline
\end{tabular}

Models are listed with formula and Akaike weight $\left(\omega_{\mathrm{i}}\right)$. Coefficient of determination, degrees of freedom and F-ratios are given in the main text. Credible intervals (in square brackets) of all predictor coefficients in these selected models do not overlap zero. This selection criterion was not met by other models within 2 AlC $\mathrm{c}_{\mathrm{c}}$ of top-ranked models (see Additional file 1: Table S1). Corticosterone response variables (ng/ml) are log-transformed to base e. Models are given for total and sex-partitioned interspecific corticosterone variation as well as for intraspecific analyses of the wedge-billed woodcreeper (G. spirurus) and olive-striped flycatcher (M. olivaceus). Abbreviated candidate variables are sampling time of day, sampling date, molt status, hemoglobin concentration, relative body mass and sampling latency. Interspecific analyses employ body mass : tarsus ratio as the relative mass variable, whereas intraspecific analyses employ a scaled mass index. ${ }^{a} \Delta \mathrm{AIC}_{\mathrm{c}}=$ 0.060 (all other models listed in this table ranked first in $\mathrm{AIC}_{\mathrm{c}}$ analyses)

woodcreeper $\left(r^{2}=0.48, \mathrm{~F}_{2,14}=6.389\right)$, with higher baseline levels found in samples of lower hemoglobin content (Fig. 2) and in those collected earlier in the day. Substantial support was not found for any model of "increase in corticosterone" in this species. Only sampling date predicted "stress-induced corticosterone" $\left(r^{2}=0.27\right.$, $F_{1,15}=5.647$ ), with individuals sampled later in the study period yielding lower levels $30 \mathrm{~min}$ post-capture.

No model for baseline corticosterone in the olivestriped flycatcher met the selection criteria. Analysis within this species identified scaled mass index as the best predictor of "increase in corticosterone" $\left(r^{2}=0.48\right.$, $\mathrm{F}_{1,8}=7.440$ ), with individuals indexed to higher relative mass yielding higher values for this corticosterone variable. This bivariate model of "increase in corticosterone" by scaled mass index remained significant for the "stress-induced corticosterone" variable $\left(r^{2}=0.47, \mathrm{~F}_{1,8}=\right.$ 7.140).

Corticosterone values from the 41 species assessed in this study are summarized in suppl. Additional file 2: Table S2 with sample sizes and phylogeny. Capture times of day for all individuals are also reported in Additional file 3: Table S3.

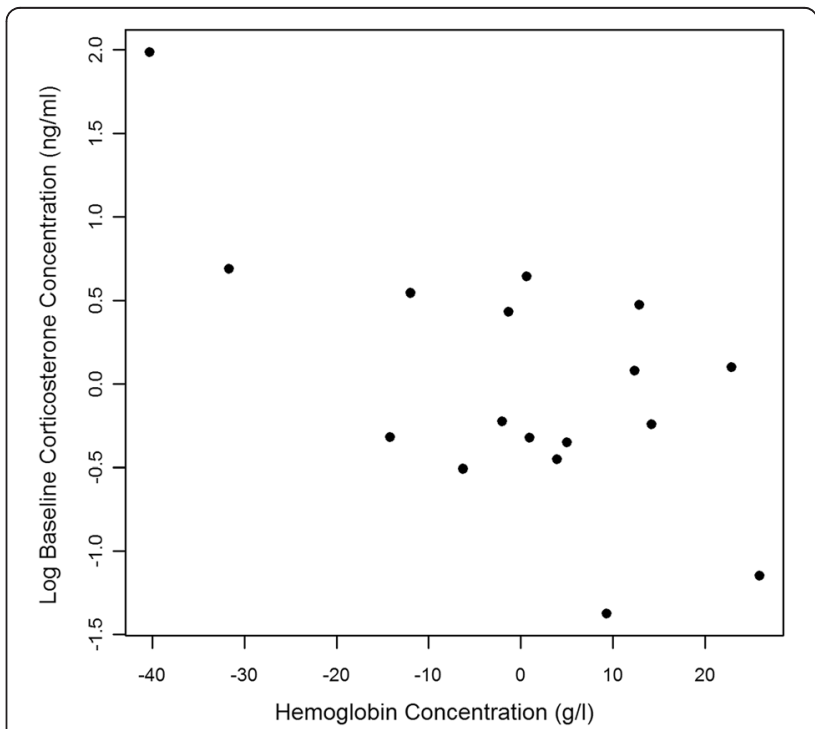

Fig. 2 Partial regression plot for baseline corticosterone and hemoglobin concentration in the wedge-billed woodcreeper. Data are presented as unstandardized residuals after controlling for sampling time of day, which best explained baseline corticosterone alongside hemoglobin concentration in $\mathrm{AlC}_{\mathrm{c}}$ model comparison $\left(r^{2}\right.$ $=0.48, F_{2,14}=6.389$; see Table 1). Log denotes the natural logarithm (to base $e$ ) 


\section{Discussion}

\section{Diurnal variation in baseline corticosterone}

Baseline corticosterone levels among 41 tropical passerine species sampled from free-living individuals generally replicated daily glucocorticoid rhythms detected in species-specific, within-individual repeated measures analyses of (primarily captive) temperate birds and mammals. As appears to be typical for mammals [4] and some non-passerines [6, 11, 56-58], diurnal baseline levels measured here were maximal at the onset of the active period and remained elevated hours thereafter. This does not oppose the dark-phase peak so far suggested for several temperate diurnal passerine species $[12,13,16,59]$, as birds were only assessed for corticosterone during daylight hours in this study. However, these results do not support the proposed rapid decline from a pre-active corticosterone peak to trough levels set early at the beginning of the light phase and maintained throughout the day [12-14].

Should avian corticosterone regulate caloric input, disposition and mobilization as do glucocorticoids in mammals [4], then our results' asynchronous baseline nadir (relative to the passerine studies cited above) may indicate distinct patterns of diurnal energy intake and demand in tropical rainforest. This biome features high productivity, stable climatic regimes and complex trophic structure [60]. Given these features, birds may indeed follow different schedules of activity because starvation and predation risk are thought to modulate foraging patterns [61]. Under predictable conditions of low starvation (higher temperatures and reduced seasonality in the tropics) [62], foraging is predicted to become less concentrated to morning and shifted later into the day [63-65]. Such dispersed (less modal) foraging time is not predicted under high predation pressure [66, 67]. However, flock formation may substantially reduce predation risk (and perception) in tropical rainforest [68, 69]. The slowed transition to trough levels of baseline corticosterone observed in this study therefore fits multiple predictions of classical avian foraging theory. Relatively short day-length near the equator (at most ten foraging hours in the sub-canopy reduced further by rainfall and mid-day heat [70]) may also give rise to prolonged foraging [66] and baseline corticosterone elevation into late morning [62]. Photoperiod-dependent altitudinal variation in diurnal foraging cycles [71] and corticosterone release $[8,16]$ side with this notion. However, independent baseline release in several other cases $[12,13,72]$ keep us from conclusions relating to photoperiod for this dataset.

In sum, our results adhere to a paradigm of timedependent baseline corticosterone secretion that emphasizes feeding routine as a basis for diel patterns in the circulating levels of this glucocorticoid, given essential bidirectional interactions between adrenocortical output, activity and metabolism [4]. Variations do not appear to correspond to taxonomic differences per se, as others have proposed for passerines in reference to non-passerines [16] and mammals [8]. Rather, they may derive from distinctions in environmental conditions such as day-length, seasonality, productivity and predation risk.

\section{Additional correlates of baseline corticosterone}

Apart from the negative correlation with sampling time of day, baseline corticosterone was also lower in molting birds of this study. This relationship persisted when analyses were conducted separately by sex and it conforms to the vast body of evidence for the down-regulation of baseline corticosterone during molt in passerines [24].

Baseline corticosterone also related to sampling date in male birds of this study. Higher levels measured at later sampling dates may follow the general progression of the breeding period into the post-hatching phase observed at our study site, during which provisioning of nestlings and fledglings likely predominates parental effort. Indeed, several recent studies present evidence for a positive effect of baseline corticosterone on foraging activity that promotes the supply of offspring with food to enhance reproductive success [73-75].

Hemoglobin concentration complemented sampling time of day as a negative correlate of baseline corticosterone in analysis within the wedge-billed woodcreeper. This negative relation reconciles poorly with other work examining links between hematocrit and corticosterone [76, 77]. However, elevated baseline corticosterone levels are often suggested to remedy poor body condition, in part by encouraging hyperphagia [78-80] when energetic status is compromised [81], such as when parents are feeding offspring. As a principal determinant of aerobic capacity, hemoglobin concentration robustly indicates energetic state [82] and is reduced when nutritional burden suppresses the formation of red blood cells [83]. It follows that low hemoglobin concentrations found in individuals of this study may have elicited the restitutive function of elevated baseline corticosterone introduced above. This perspective accommodates our data on birds captured primarily during breeding (i.e. compromised energetic condition). It also complies with theory that baseline corticosterone mediates trade-offs between parental investment and self-maintenance by regulating foraging activity [84].

Lastly, baseline corticosterone levels increased with sampling latency in interspecific analyses of samples taken within 3 min of capture. Initiation of stress-related corticosterone release has been specified to $1.5-2 \mathrm{~min}$ post-capture in other passerines before $[85,86]$. Nevertheless, most studies continue to designate baseline levels based on the assumption that $3 \mathrm{~min}$ represent stressindependent release [48]. 
Correlates of corticosterone release in response to stress Evidence from the wild of time-dependent adrenocortical response to stress in birds remains elusive, as neither "increase in corticosterone" nor "stress-induced corticosterone" exhibited any diurnal rhythm in this study. To the contrary, our results confirm evidence for the downregulation of the avian stress response during molt [24]. The negative interspecific "stress-induced corticosterone" relationship to molt status scaled up in analysis within males and also extended to the "increase in corticosterone" variable in this sex. Per definition (see Methods), "increase in corticosterone" is inversely related to baseline corticosterone levels. As such, its sustained correlation to molt status in the face of a parallel molt relationship at baseline levels emphasizes the weight of the molt effect on corticosterone release in response to stress. These results advocate the hypothesis of corticosterone down-regulation selected to preclude proteolytic action at stress-related concentrations during feather formation [87]. Such down-regulation should be particularly critical for molting males, for which plumage quality can come to dictate mating success [88].

"Increase in corticosterone" and "stress-induced corticosterone" also related positively to sampling date in our interspecific analyses of males, and a trend for the former was also present in females. As suggested for baseline corticosterone, these elevations may parallel the observed transition from incubation to post-hatching reproductive stages. Brooding effort in neo-tropical passerines often decreases with increased post-hatching provisioning (e.g. [89]) when offspring become more independent in thermoregulation but remain dependent for food acquisition [90] such that parents may afford a strong stress response to enhance short-term survival probability during increased foraging effort. Interestingly, sampling date correlated negatively with "stress-induced corticosterone" in the wedge-billed woodcreeper, a cavity-nesting bird [91]. Given lower levels of nest predation [92] and microclimate variation [93], cavity-nesters may shift high levels of nest attentiveness into later stages of the reproductive cycle [94] with associated alterations in the magnitude of their stress response.

Lastly, greater corticosterone release in response to stress was found in olive-striped flycatcher individuals with higher scaled mass indices. These indices indicate size of fat and protein reserves [52]. Their positive relation to "stress-induced corticosterone" (as well as the reduction of interspecific stress-related levels during molt, a process of high energetic cost [95]) therefore adds to accumulating evidence that an elevated energetic state is accompanied by greater stress-related corticosterone secretion [96-98]. This evidence sides with the hypothesis that the advantages of a strong adrenocortical response are most affordable when surplus fat and muscle substrates can be resigned to gluconeogenesis with little compromise to other vital physiological processes $[99,100]$.

\section{Conclusions}

Our results indicate diurnal variation in baseline corticosterone release in free-living tropical forest birds. This rhythmicity appears robust enough to surface in analyses from observations pooled across a most diverse array of passerine species. It does not point to elementary departures from other taxa in temporal patterns and regulation of passerine corticosterone release. The observed baseline relationships presumably issue from bi-directional effects of corticosterone secretion and metabolic state as in other animals. Moreover, life-history state may set bounds on this condition-dependent corticosterone release at both baseline and stress-related levels. Modest alterations in corticosterone reaction to environmental and intrinsic signals are nevertheless likely to eventuate from ecological disparities among biomes of different latitudes. This study suggests certain responses to time of day and other variables to be tuned to life in highproductivity, relatively aseasonal tropical rainforest.

In practical terms, the results presented here offer several lines of orientation for improved methods in tropical avian field endocrinology. These include bearings for appropriate sampling schedule at multiple time scales (relating to season, time of day and latency) and variable parameterization (e.g. caution with relative measures of corticosterone response) as well as a range of corticosterone concentrations to be anticipated in tropical rainforest passerines. We hope that this information accelerates progress in grasping corticosterone's complex interactions with avian physiology and behavior in the tropics and beyond.

\section{Ethics approval and consent to participate}

This research was authorized by Ministerio del Ambiente del Ecuador in accordance to national guidelines.

\section{Consent for publication}

Not applicable.

\section{Additional files}

Additional file 1: Table S1. Baseline corticosterone (y1), "increase in corticosterone" (y2) and "stress-induced corticosterone" (y3) models from $\mathrm{AlC}_{\mathrm{c}}$ analyses. (PDF $344 \mathrm{~kb}$ )

Additional file 2: Table S2. Species median values with sample sizes for baseline and "stress-induced corticosterone" concentrations. (PDF 286 kb)

Additional file 3: Table S3. Time of capture for 95 individuals (ID) of 41 species analyzed for baseline and stress-related corticosterone concentrations. (PDF $211 \mathrm{~kb}$ )

Competing interests

The authors declare that they have no competing interests. 


\section{Authors' contributions}

PS conceived the study. WG and EB refined its design. PS collected the data. PS performed laboratory procedures. PS and WG statistically analyzed results. PS wrote the manuscript. WG and EB provided revisions. All authors read and approved the final manuscript.

\section{Acknowledgements}

We thank J. Wolinska and R. de Bruijn for advice on study methods and design, C. Morochz for expertise and companionship in the field, all others at Mashpi Lodge for tremendous generosity and logistical infrastructure, M. Trappschuh for assistance with hormone analyses, Universidad Tecnológica Indoamérica (Quito) for institutional support in obtaining permits and field equipment and Ministerio del Ambiente del Ecuador for authorizing this research (No. 19-2014-IC-FAU-DPAP-MA).

\section{Funding}

Max-Planck-Gesellschaft funded laboratory analyses. PROSA-LMU and Mashpi Lodge funded field research.

\section{Author details}

'Institute of Biodiversity, Animal Health and Comparative Medicine, University of Glasgow, Graham Kerr Building, Glasgow G12 8QQ, UK. ${ }^{2}$ Centro para la Investigación de la Biodiversidad y Cambio Climático, Universidad Tecnológica Indoamérica, Machala y Sabanilla, Cotocollao, Quito, Ecuador. ${ }^{3}$ Abteilung für Verhaltensneurobiologie, Max-Planck-Institut für Ornithologie, Eberhard-Gwinner-Str. 6a, D-82319 Seewiesen, Germany.

\section{Received: 7 February 2016 Accepted: 28 April 2016}

\section{Published online: 04 May 2016}

\section{References}

1. Busch DS, Hayward LS. Stress in a conservation context: a discussion of glucocorticoid actions and how levels change with conservation-relevant variables. Biol Conserv. 2009;142:2844-53.

2. Sapolsky RM, Romero LM, Munck AU. How do glucocorticoids influence stress responses? Integrating permissive, suppressive, stimulatory, and preparative actions. Endocr Rev. 2000:21:55-89.

3. Landys MM, Ramenofsky M, Wingfield JC. Actions of glucocorticoids at a seasonal baseline as compared to stress-related levels in the regulation of periodic life processes. Gen Comp Endocrinol. 2006;148:132-49.

4. Dallman MF, Strack AM, Akana SF, Bradbury MJ, Hanson ES, Scribner KA, et al. Feast and famine: critical role of glucocorticoids with insulin in daily energy flow. Front Neuroendocrinol. 1993;14:303-47.

5. Spiga F, Walker JJ, Terry JR, Lightman SL. HPA axis-rhythms. Compr Physiol. 2014;4:1273-98.

6. Boissin J. Circadian rhythm of adrenal cortex function, general activity and internal temperature in quails. Bull Biol Fr Belg. 1969;103:305-12.

7. Hazard D, Couty M, Faure JM, Guémené D. Daily and photoperiod variations of hypothalamic-pituitary-adrenal axis responsiveness in Japanese quail selected for short or long tonic immobility. Poult Sci. 2005:84:1920-5.

8. Romero LM, Rich EL. Photoperiodically-induced changes in hypothalamicpituitary-adrenal axis sensitivity in captive house sparrows (Passer domesticus). Comp Biochem Physiol A Mol Integr Physiol. 2007;147:562-8.

9. Holmes WN, Phillips JG. The adrenal cortex of birds. In: Chester-Jones I Henderson IW, editors. General, Comparative and Clinical Endocrinology of the Adrenal Cortex. London: Academic Press; 1976. p. 293-420.

10. Johnson AL, van Tienhoven A. Plasma concentrations and corticosterone relative to photoperiod, oviposition, and ovulation in the domestic hen Gen Comp Endocrinol. 1981:43:10-6.

11. Westerhof I, Mol JA, Van den Brom WE, Lumeij JT, Rijnberk A. Diurnal rhythms of plasma corticosterone concentrations in racing pigeons (Columba livia domestica) exposed to different light regimens, and the influence of frequent blood sampling. Avian Dis. 1994;38:428-34.

12. Breuner CW, Wingfield JC, Romero LM. Diel rhythms of basal and stressinduced corticosterone in a wild, seasonal vertebrate, Gambel's whitecrowned sparrow. J Exp Zool. 1999:284:334-42.

13. Romero LM, Remage-Healey L. Daily and seasonal variation in response to stress in captive starlings (Sturnus vulgaris): corticosterone. Gen Comp Endocrinol. 2000;119:52-9.

14. Tarlow EM, Hau M, Anderson DJ, Wikelski M. Diel changes in plasma melatonin and corticosterone concentrations in tropical Nazca boobies
(Sula granti) in relation to moon phase and age. Gen Comp Endocrinol. 2003:133:297-304

15. Goymann W, Trappschuh M. Seasonal and diel variation of hormone metabolites in European stonechats: on the importance of high signal-tonoise ratios in noninvasive hormone studies. J Biol Rhythms. 2011;26:44-54.

16. Rich EL, Romero LM. Daily and photoperiod variations of basal and stressinduced corticosterone concentrations in house sparrows (Passer domesticus). J Comp Physiol B. 2001;171:543-7.

17. Romero LM. Seasonal changes in hypothalamic-pituitary-adrenal axis sensitivity in free-living house sparrows (Passer domesticus). Gen Comp Endocrinol. 2006;149:66-71.

18. Blas J. Stress in birds. In: Scanes CG, editor. Sturkie's Avian Physiology. 6th ed. London: Academic Press; 2015. p. 769-810.

19. Marra PP, Lampe KT, Tedford BL. Plasma corticosterone levels in two species of Zonotrichia sparrows under captive and free-living conditions. Wilson Bull. 1995;107:296-305

20. Romero LM, Wingfield JC. Alterations in hypothalamic-pituitary-adrenal function associated with captivity in Gambel's white-crowned sparrows (Zonotrichia leucophrys gambelii). Comp Biochem Physiol B Biochem Mol Biol. 1999;122:13-20

21. Wingfield JC, Hegner RE, Dufty AM, Ball GF. The "challenge hypothesis": theoretical implications for patterns of testosterone secretion, mating systems, and breeding strategies. Am Nat. 1990;136:829-46.

22. Martin LB, Rubenstein DR. Stress hormones in tropical birds: patterns and future directions. Ornitol Neotropical. 2008;19:207-18.

23. Wingfield JC, Hunt KE. Arctic spring: hormone-behavior interactions in a severe environment. Comp Biochem Physiol B Biochem Mol Biol. 2002;132: 275-86.

24. Romero LM. Seasonal changes in plasma glucocorticoid concentrations in free-living vertebrates. Gen Comp Endocrinol. 2002;128:1-24.

25. Martin TE. A new view of avian life-history evolution tested on an incubation paradox. Proc R Soc B Biol Sci. 2002;269:309-16.

26. Finch CE, Rose MR. Hormones and the physiological architecture of life history evolution. Q Rev Biol. 1995;70:1-52.

27. Eikenaar C, Husak J, Escallón C, Moore IT, Angilletta AEM, Bronstein EJL. Variation in testosterone and corticosterone in amphibians and reptiles: relationships with latitude, elevation, and breeding season length. Am Nat. 2012;180:642-54.

28. Hau M. Regulation of male traits by testosterone: implications for the evolution of vertebrate life histories. Bioessays. 2007;29:133-44.

29. Schwabl H, Palacios MG, Martin TE, Adkins-Regan AEE, Whitlock EMC. Selection for rapid embryo development correlates with embryo exposure to maternal androgens among passerine birds. Am Nat. 2007;170:196-206.

30. Martin TE, Schwabl H. Variation in maternal effects and embryonic development rates among passerine species. Philos Trans R Soc Lond B Biol Sci. 2008;363:1663-74.

31. Bókony V, Lendvai AZ, Liker A, Angelier F, Wingfield JC, Chastel O. Stress response and the value of reproduction: are birds prudent parents? Am Nat. 2009:173:589-98.

32. Hau M, Ricklefs RE, Wikelski M, Lee KA, Brawn JD. Corticosterone, testosterone and life-history strategies of birds. Proc R Soc Lond B Biol Sci. 2010;277:3203-12.

33. Bonier F, Martin PR, Moore IT, Wingfield JC. Do baseline glucocorticoids predict fitness? Trends Ecol Evol. 2009;24:634-42.

34. Madliger $\mathrm{CL}$, Love OP. The need for a predictive, context-dependent approach to the application of stress hormones in conservation. Conserv Biol J Soc Conserv Biol. 2014:28:283-7.

35. Dickens JM, Romero LM. A consensus endocrine profile for chronically stressed wild animals does not exist. Gen Comp Endocrinol. 2013;191: $177-89$.

36. Silverin B, Arvidsson B, Wingfield J. The adrenocortical responses to stress in breeding willow warblers Phylloscopus trochilus in Sweden: effects of latitude and gender. Funct Ecol. 1997;11:376-84

37. Angelier F, Wingfield JC, Weimerskirch $\mathrm{H}$, Chastel $\mathrm{O}$. Hormonal correlates of individual quality in a long-lived bird: a test of the "corticosterone-fitness hypothesis.". Biol Lett. 2010;6:846-9.

38. Myers NM, Mittermeier RA, Mittermeier CG, da Fonseca GAB, Kent J. Biodiversity hotspots for conservation priorities. Nature. 1999:403:853-8.

39. Quillfeldt P, Poisbleau M, Chastel O, Masello JF. Corticosterone in thin-billed prion Pachyptila belcheri chicks: diel rhythm, timing of fledging and nutritional stress. Naturwissenschaften. 2007;94:919-25. 
40. Wingfield JC, Hunt K, Breuner C, Dunlap K, Fowler GS, Freed L, Lepson J. Environmental stress, field endocrinology, and conservation biology. In: Clemmons JR, Buchholz R, editors. Behavioral Approaches to Conservation in the Wild. Cambridge: Cambridge University Press; 1997. p. 95-131.

41. Polo V, Bautista LM. Daily routines of body mass gain in birds: 1. an exponential model. Anim Behav. 2006;72:503-16.

42. Mittermeier RA, Turner WR, Larsen FW, Brooks TM, Gascon C. Global biodiversity conservation: the critical role of hotspots. In: Zachos FE, Habel JC, editors. Biodiversity Hotspots: Distribution and Protection of Conservation Priority Areas. Berlin: Springer-Verlag; 2011. p. 3-22.

43. Hijmans RJ, Cameron SE, Parra JL, Jones PG, Jarvis A. Very high resolution interpolated climate surfaces for global land areas. Int J Climatol. 2005;25: 1965-78.

44. NOAA ESRL Global Monitoring Division. NOAA solar calculator. [https:// www.esrl.noaa.gov/gmd/grad/solcalc/index.html].

45. Greeney HF, Nunnery T. Notes on the breeding of north-west Ecuadorian birds. Bull Br Ornithol Club. 2006;126:38-45.

46. Carrasco B, Berg KS, Litz J, Cook A, Karubian J. Avifauna of the Mache Chindul ecological reserve, northwest Ecuador. Ornitol Neotropical. 2013;24: 321-34

47. Solano-Ugalde A, Arcos-Torres A, Greeney HF. Additional breeding records for selected avian species in northwest Ecuador. Boletín SAO. 2007;17:17-25

48. Wingfield JC, Smith JP, Farner DS. Endocrine responses of white-crowned sparrows to environmental stress. Condor. 1982;84:399-409.

49. Goymann W, Schwabl I, Trappschuh M, Hau M. Use of ethanol for preserving steroid and indoleamine hormones in bird plasma. Gen Comp Endocrinol. 2007;150:191-5.

50. R Core Team. R. A language and environment for statistical computing. Vienna: R Foundation for Statistical Computing; 2015.

51. Gelman A, Su Y-S. arm: Data analysis using regression and multilevel/ hierarchical models. R package version 1.8-6. 2015. [https://cran.r-project. org/web/packages/arm/index.html].

52. Peig J, Green AJ. New perspectives for estimating body condition from mass/length data: the scaled mass index as an alternative method. Oikos. 2009;118:1883-91.

53. Burnham KP, Anderson DR. Model Selection and Multimodel Inference: A Practical Information-Theoretic Approach. 2nd ed. New York: SpringerVerlag; 2002.

54. Paradis E, Blomberg S, Bolker B, Claude J, Cuong HS, Desper R, Didier G, Durand B, Dutheil J, Gascuel O, Heibl C, Ives A, Lawson D, Lefort V, Legendre P, Lemon J, McCloskey R, Nylander J, Opgen-Rhein R, Popescu AA, Royer-Carenzi M, Schliep K, Strimmer K, de Vienne D. ape: Analyses of phylogenetics and evolution in $\mathrm{R}$ language. $\mathrm{R}$ package version 3.4. 2015 [https://cran.r-project.org/web/packages/ape/index.html].

55. Jetz W, Thomas GH, Joy JB, Redding DW, Hartmann K, Mooers AO. Global distribution and conservation of evolutionary distinctness in birds. Curr Biol. 2014:24:919-30

56. Joseph MM, Meier AH. Daily rhythms of plasma corticosterone in the common pigeon, Columba livia. Gen Comp Endocrinol. 1973;20: 326-30.

57. Kovács K, Péczely P, Pethes G. Daily fluctuations of peripheral metabolism of corticosterone in male Japanese quails: a dynamic approach to the development of plasma corticosterone daily rhythm. Comp Biochem Physiol A Physiol. 1983;75:467-9.

58. Lauber JK, Vriend J, Oishi T. Plasma corticosterone in chicks reared under several lighting schedules. Comp Biochem Physiol A Physiol. 1987;86:73-8.

59. Dusseau JW, Meier AH. Diurnal and seasonal variations of plasma adrenal steroid hormone in the white-throated sparrow, Zonotrichia albicollis. Gen Comp Endocrinol. 1971;16:399-408.

60. Levins R. Evolution in Changing Environments: Some Theoretical Explanations. Princeton: Princeton University Press; 1968.

61. Brodin A. Theoretical models of adaptive energy management in small wintering birds. Philos Trans R Soc B Biol Sci. 2007;362:1857-71.

62. Rogers $C M$, Heath-Coss R. Effect of experimentally altered food abundance on fat reserves of wintering birds. J Anim Ecol. 2003;72:822-30.

63. Brandt MJ, Cresswell W. Diurnal foraging routines in a tropical bird, the rock finch Lagonosticta sanguinodorsalis: how important is predation risk? J Avian Biol. 2009:40:90-4.

64. Olsson O, Wiktander U, Nilsson SG. Daily foraging routines and feeding effort of a small bird feeding on a predictable resource. Proc Biol Sci. 2000; 267:1457-61.
65. MacLeod R, Barnett P, Clark JA, Cresswell W. Body mass change strategies in blackbirds Turdus merula: the starvation-predation risk trade-off. J Anim Ecol. 2005;74:292-302.

66. McNamara JM, Houston Al, Lima SL. Foraging routines of small birds in winter: a theoretical investigation. J Avian Biol. 1994;25:287-302.

67. Macleod R, Gosler AG, Cresswell W. Diurnal mass gain strategies and perceived predation risk in the great tit Parus major. J Anim Ecol. 2005;74: 956-64.

68. Thiollay J-M. Pressions de prédation comparées sur trois groupements d'oiseaux en forêt tropicale. Le Gerfaut. 1984;74:209-25.

69. Thiollay J-M. Frequency of mixed species flocking in tropical forest birds and correlates of predation risk: an intertropical comparison. J Avian Biol. 1999:30:282-94.

70. Bongers F, Charles-Dominique P, Forget P-M, Théry M. Nouragues: Dynamics and Plant-Animal Interactions in a Neotropical Rainforest. Dordrecht: Springer Netherlands; 2013.

71. Polo V, Carrascal LM, Metcalfe NB. The effects of latitude and day length on fattening strategies of wintering coal tits Periparus ater (L.): a field study and aviary experiment. J Anim Ecol. 2007;76:866-72.

72. Pravosudov W, Kitaysky AS, Saldanha CJ, Wingfield JC, Clayton NS. The effect of photoperiod on adrenocortical stress response in mountain chickadees (Poecile gambeli). Gen Comp Endocrinol. 2002;126:242-8.

73. Angelier F, Bost C-A, Giraudeau M, Bouteloup G, Dano S, Chastel O. Corticosterone and foraging behavior in a diving seabird: the Adélie penguin, Pygoscelis adeliae. Gen Comp Endocrinol. 2008;156:134-44.

74. Miller DA, Vleck CM, Otis DL. Individual variation in baseline and stressinduced corticosterone and prolactin levels predicts parental effort by nesting mourning doves. Horm Behav. 2009;56:457-64.

75. Crossin GT, Trathan PN, Phillips RA, Gorman KB, Dawson A, Sakamoto KQ, et al. Corticosterone predicts foraging behavior and parental care in macaroni penguins. Am Nat. 2012;180:E31-41.

76. Buehler DM, Vézina F, Goymann W, Schwabl I, Versteegh M, Tieleman BI, Piersma T. Independence among physiological traits suggests flexibility in the face of ecological demands on phenotypes. J Evol Biol. 2012;25: 1600-13.

77. Grunst ML, Rotenberry JT, Grunst AS. Variation in adrenocortical stress physiology and condition metrics within a heterogeneous urban environment in the song sparrow Melospiza melodia. J Avian Biol. 2014;45: 574-83.

78. Wingfield JC, Maney DL, Breuner CW, Jacobs JD, Lynn S, Ramenofsky M, Richardson RD. Ecological bases of hormone-behavior interactions: the "emergency life history stage". Am Zool. 1998;38:191-206.

79. Landys MM, Ramenofsky M, Guglielmo CG, Wingfield JC. The low-affinity glucocorticoid receptor regulates feeding and lipid breakdown in the migratory Gambel's white-crowned sparrow Zonotrichia leucophrys gambelii. J Exp Biol. 2004;207:143-54.

80. Holberton RL, Wilson CM, Hunter MJ, Cash WB, Sims CG. The role of corticosterone in supporting migratory lipogenesis in the dark-eyed Junco, Junco hyemalis: a model for central and peripheral regulation. Physiol Biochem Zool. 2007:80:125-37.

81. Astheimer LB, Buttemer WA, Wingfield JC. Interactions of corticosterone with feeding, activity and metabolism in passerine birds. Ornis Scand. 1992; 23:355-65.

82. Minias $P$. The use of haemoglobin concentrations to assess physiological condition in birds: a review. Conserv Physiol. 2015;3:cov007.

83. Jones PJ. Haematocrit values of breeding red-billed queleas Quelea quelea (Aves: Ploceidae) in relation to body condition and thymus activity. J Zool. 1983;201:217-22.

84. Thierry A-M, Ropert-Coudert $Y$, Raclot T. Elevated corticosterone levels decrease reproductive output of chick-rearing Adélie penguins but do not affect chick mass at fledging. Conserv Physiol. 2013;1:cot007.

85. Dawson A, Howe PD. Plasma corticosterone in wild starlings (Sturnus vulgaris) immediately following capture and in relation to body weight during the annual cycle. Gen Comp Endocrinol. 1983;51:303-8.

86. Romero LM, Reed JM. Collecting baseline corticosterone samples in the field: is under 3 min good enough? Comp Biochem Physiol A Mol Integr Physiol. 2005; 140:73-9.

87. Romero LM, Strochlic D, Wingfield JC. Corticosterone inhibits feather growth: potential mechanism explaining seasonal down regulation of corticosterone during molt. Comp Biochem Physiol A Mol Integr Physiol. 2005;142:65-73. 
88. Pryke SR, Andersson S. Experimental evidence for female choice and energetic costs of male tail elongation in red-collared widowbirds. Biol J Linn Soc. 2005;86:35-43.

89. Goulding W, Martin TE. Breeding biology of the golden-faced tyrannulet (Zimmerius chrysops) in Venezuela. Wilson J Ornithol. 2010;122:689-98.

90. Wetherell E, Greeney HF, Port J. Breeding biology of speckled hummingbird Adelomyia melanogenys in eastern Ecuador. Cotinga. 2015;37:43-6.

91. Ridgely RS, Tudor G. The Birds of South America: Vol. II, The Suboscine Passerines. Austin: University of Texas Press; 1994.

92. Fontaine JJ, Martel M, Markland HM, Niklison AM, Decker KL, Martin TE. Testing ecological and behavioral correlates of nest predation. Oikos. 2007; 116:1887-94.

93. Martin TE, Li P. Life history traits of open- vs. cavity-nesting birds. Ecology. 1992;73:579-92.

94. Martin TE, Ghalambor CK, Byers AEJA, Gowaty PA. Males feeding females during incubation. I. Required by microclimate or constrained by nest predation? Am Nat. 1999;153:131-9.

95. DesRochers DW, Reed JM, Awerman J, Kluge JA, Wilkinson J, van Griethuijsen LI, et al. Exogenous and endogenous corticosterone alter feather quality. Comp Biochem Physiol A Mol Integr Physiol. 2009;152:46-52.

96. Jenni L, Jenni-Eiermann S, Spina F, Schwabl H. Regulation of protein breakdown and adrenocortical response to stress in birds during migratory flight. Am J Physiol Regul Integr Comp Physiol. 2000;278:R1182-9.

97. Lynn SE, Breuner CW, Wingfield JC. Short-term fasting affects locomotor activity, corticosterone, and corticosterone binding globulin in a migratory songbird. Horm Behav. 2003:43:150-7.

98. Schoech SJ, Bowman R, Bridge ES, Boughton RK. Baseline and acute levels of corticosterone in Florida scrub-jays (Aphelocoma coerulescens): effects of food supplementation, suburban habitat, and year. Gen Comp Endocrinol. 2007:154:150-60

99. Fokidis HB, Hurley L, Rogowski C, Sweazea K, Deviche P. Effects of captivity and body condition on plasma corticosterone, locomotor behavior, and plasma metabolites in curve-billed thrashers. Physiol Biochem Zool. 2011;84: 595-606.

100. Scanes CG. Perspectives on the endocrinology of poultry growth and metabolism. Gen Comp Endocrinol. 2009;163:24-32.

\section{Submit your next manuscript to BioMed Central and we will help you at every step:}

- We accept pre-submission inquiries

- Our selector tool helps you to find the most relevant journal

- We provide round the clock customer support

- Convenient online submission

- Thorough peer review

- Inclusion in PubMed and all major indexing services

- Maximum visibility for your research

Submit your manuscript at www.biomedcentral.com/submit

) Biomed Central 